\title{
Therapeutic Feasibility of Full Endoscopic Decompression in One- to Three-Level Lumbar Canal Stenosis via a Single Skin Port Using a New Endoscopic System, Percutaneous Stenoscopic Lumbar Decompression
}

\author{
Kang Taek Lim, Han Ga Wi Nam, Soo Beom Kim, Hyung Suk Kim, Jin Soo Park, Chun-Kun Park
}

Department of Neurosurgery, Good Doctor Teun Teun Hospital, Anyang, Korea

\begin{abstract}
Study Design: This retrospective study involved 450 consecutive cases of degenerative lumbar stenosis treated with percutaneous stenoscopic lumbar decompression (PSLD).

Purpose: We determined the feasibility of PSLD for lumbar stenosis at single and multiple levels (minimum 1-year follow-up) by image analysis to observe postoperative widening of the vertebral canal in the area.

Overview of Literature: The decision not to perform an endoscopic decompression might be due to the surgeon being uncomfortable with conventional microscopic decompression or unfamiliar with endoscopic techniques or the unavailability of relevant surgical tools to completely decompress the spinal stenosis.

Methods: The decompressed canal was compared between preoperative controls and postoperative treated cases. Data on operative results, including length of stay, operative time, and surgical complications, were analyzed. Patients were assessed clinically on the basis of the Visual Analog Scale (VAS) score for the back and legs and using the Oswestry Disability Index (ODI).

Results: Postoperative magnetic resonance imaging revealed that PSLD increased the canal cross-sectional area by $52.0 \%$ compared with the preoperative area at the index segment $(p<0.001)$ and demonstrated minimal damage to the normal soft tissues including muscles and the extent of removed normal bony tissues. Mean improvements in VAS score and ODI were $4.0(p<0.001)$ and $40 \%$ $(p<0.001)$, respectively.

Conclusions: PSLD could be an alternative to microscopic or microendoscopic decompression with various advantages in the surgical management of lumbar stenosis.
\end{abstract}

Keywords: Spinal stenosis; Percutanous stenoscopic lumbar decompression; Minimally invasive spine surgery; Decompression

\section{Introduction}

Degenerative changes in spine structures, including discs, ligamentum flavum, and facet, lead to spinal stenosis [1,2]. Spinal stenosis is a common indication for spinal surgery in patients $>65$ years old $[3,4]$. As the elderly population is

Received Sep 8, 2018; Revised Oct 7, 2018; Accepted Oct 7, 2018

Corresponding author: Kang Taek Lim

Department of Neurosurgery, Good Doctor Teun Teun Hospital, 775 Gyeongsu-Daero Dongan-gu, Anyang 14041, Korea

Tel: +82-31-8086-8357, Fax: +82-2-534-4799, E-mail: limkat@hanmail.net 
constantly increasing in developed countries, the incidence of spinal stenosis, which occurs mainly in aged people, also is expected to increase [5]. Spinal stenosis can develop in three interconnected structures in the spine. Monoportal percutaneous stenoscopic lumbar decompression (PSLD) holds the potential for managing monolevel and multilevel spinal stenosis, central canals, lateral recesses, and foramen [6]. Traditionally, lumbar spinal stenosis is decompressed with open laminectomy, which involves removing the spinous process, lamina, and posterior musculoligamentous complex. However, open laminectomy is associated with potential postoperative complications, including spinal instability, which may result in the future need for spinal fusion [7]. Until now, several kinds of minimally invasive spine surgery (MISS) techniques have been applied for management of spinal stenosis, the most popular of which is microscopic decompression. Recently, microendoscopic decompression (MED) has become a representative MISS technique to treat spinal stenosis $[8,9]$. Meanwhile, a spinal endoscopic system was designed for and applied to lumbar disc herniation mainly through a transforaminal route, but recently, this technique faced various challenges and obstacles, including certain pathologies not accessible via the transforaminal approach [10]. Most challenges have been overcome by new techniques and approaches; however, most suggested means are time-consuming, require experienced hands, and have a very steep learning curve for surgeons. Few reports exist regarding postoperative changes in certain clinical parameters, such as the Visual Analog Scale (VAS) and Oswestry Disability Index (ODI), in spinal stenosis following endoscopic decompression via an interlaminar approach using conventional endoscopic systems $[11,12]$. Most interlaminar approach studies involved a small number of patients, and few surgeons have applied the technique in spinal stenosis. The decision not to perform an endoscopic decompression might be due to the surgeon being uncomfortable with conventional microscopic decompression or unfamiliar with endoscopic techniques and the unavailability of relevant surgical tools to fully decompress the spinal stenosis. PSLD could be a means to overcome the obstacles encountered with the transforaminal endoscopic technique. PSLD leads to decompression of the central and lateral recess spinal stenosis and removes any type of herniated disc simultaneously via a translaminar approach using the stenoscope. Furthermore, a new technique is displayed, which decompresses 2-3 levels of the stenosis concomitantly via uniportal ac- cess through one skin incision in the lumbar spine (jumping technique). We determined the feasibility of PSLD for lumbar stenosis at single and multiple levels (minimum 1-year follow-up) by image analysis to observe postoperative widening of the vertebral canal in the stenotic area.

\section{Materials and Methods}

\section{Patients}

This retrospective study was exempted from institutional review board of Good Doctor Teun Teun Hospital. And the informed consent was waived. We studied 450 lumbar stenosis patients (532 disc levels; 254 males, 196 females;

Table 1. Demographic data of 450 patients (532 disc level) with degenerative lumbar stenosis

\begin{tabular}{|c|c|}
\hline Characteristic & Value \\
\hline Age (yr) & $67.1 \pm 12$ \\
\hline Sex (male:female) & 254:196 \\
\hline Duration of symptoms (mo) & $22.3 \pm 21.4$ \\
\hline \multicolumn{2}{|l|}{ Decompression level $(n=450)$} \\
\hline 1 Level & 388 \\
\hline 2 Level & 42 \\
\hline 3 Level & 30 \\
\hline \multicolumn{2}{|c|}{ Decompression segment $(n=532)$} \\
\hline \multicolumn{2}{|c|}{1 Level (n=388) } \\
\hline L1-2 & 10 \\
\hline L2-3 & 28 \\
\hline L3-4 & 52 \\
\hline$\lfloor 4-5$ & 139 \\
\hline L5-S1 & 159 \\
\hline \multicolumn{2}{|l|}{2 Level (n=84) } \\
\hline L1-2 & 4 \\
\hline L2-3 & 12 \\
\hline L3-4 & 20 \\
\hline$\llcorner 4-5$ & 30 \\
\hline L5-S1 & 18 \\
\hline \multicolumn{2}{|l|}{3 Level $(n=60)$} \\
\hline L1-2 & 3 \\
\hline $\mathrm{L} 2-3$ & 10 \\
\hline L3-4 & 20 \\
\hline$\lfloor 4-5$ & 17 \\
\hline L5-S1 & 10 \\
\hline
\end{tabular}

Values are presented as mean \pm standard deviation or number. 
mean age, 67.1 years) with central and/or lateral recess stenosis who underwent consecutive bilateral decompression using PSLD at a spine-specialized hospital between April 2016 and December 2016. All patients had symptoms of back pain and bilateral neurogenic claudication with lumbar stenosis (magnetic resonance imaging [MRI] evidence) and underwent at least 3 months of conservative treatment. Patients presenting with foraminal stenosis, combined lesion with disc herniation, or more than grade 1 degenerative spondylolisthesis were excluded from this study. The level of stenosis was L4-5 in 139 patients, L5$\mathrm{S} 1$ in 159, L3-4 in 52, L2-3 in 28, L1-2 in 10, 2-level in 42 , and 3-level in 20 (Table 1). The cross-sectional area of the spinal canal at an index segment on lumbar MRI was measured by a foreign clinical fellow, who had no access to information about the examinee. The fellow compared the decompressed canal between the preoperative controls and postoperative treated patients. In each patient, three slices were obtained from the vertebral canal at the index level (upper, middle, and lower levels) at every $6 \mathrm{~mm}$ interval (Fig. 1). Other data on operative results, including length of stay, operative time, and surgical complications, were analyzed. A total of 250 patients (118 males, 132 females) were clinically assessed on the basis of the VAS score for the back and legs preoperatively, and at 1 week, and 3 and 12 months postoperatively, and on the ODI preoperatively and at 3 and 12 months postoperatively.

\section{The specifications of endoscopic instruments}

We evaluated the size of the lamina and facet angle in patients with lumbar stenosis to build up a baseline data set to design an optimal endoscopic procedure for lumbar stenosis. Endoscopy with a suitable outer diameter instrument was expected to minimize further iatrogenic muscle injury and avoid destabilization of bony structures while approaching the lesion. According to mean values
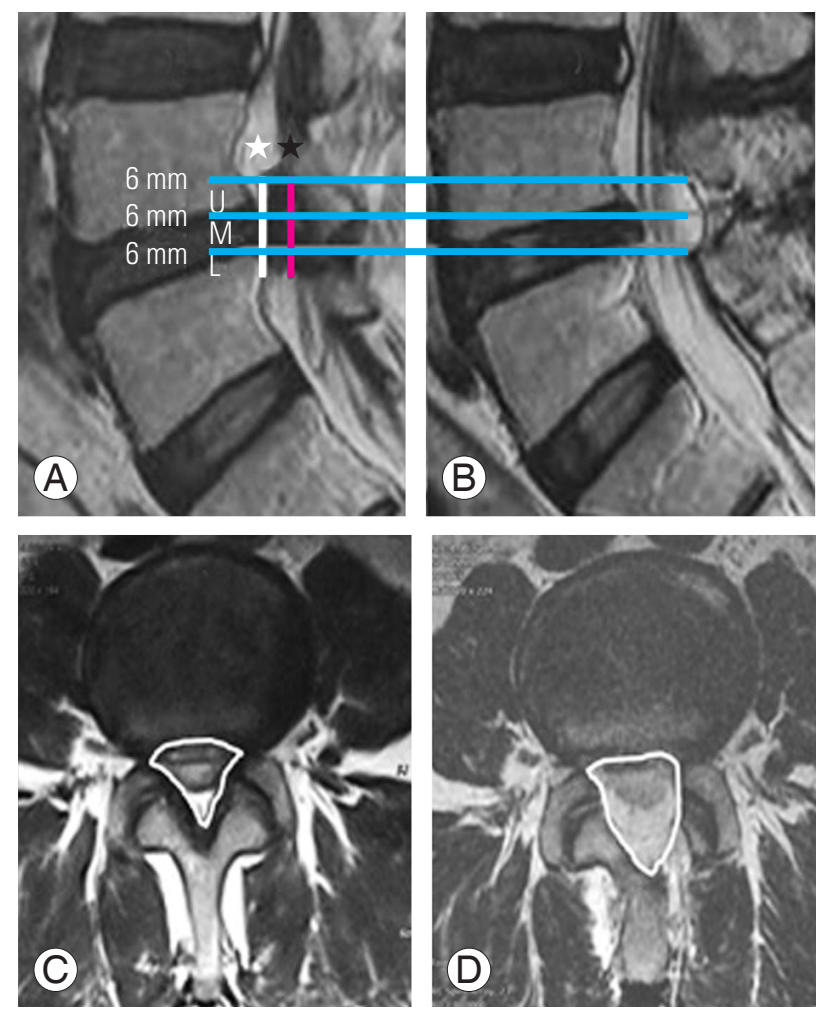

Fig. 1. (A, B) Preoperative and postoperative sagittal $6 \mathrm{~mm}$ cutting from upper to lower ligamentum flavum MRI. (C, D) Preoperative and postoperative cross-sectional area. $\star$ (black), decompressed segment; 2 (white), a calculated segment with $6 \mathrm{~mm}$ height; $U$, upper cross-sectional segment; $M$, middle segment; L, lower segment.
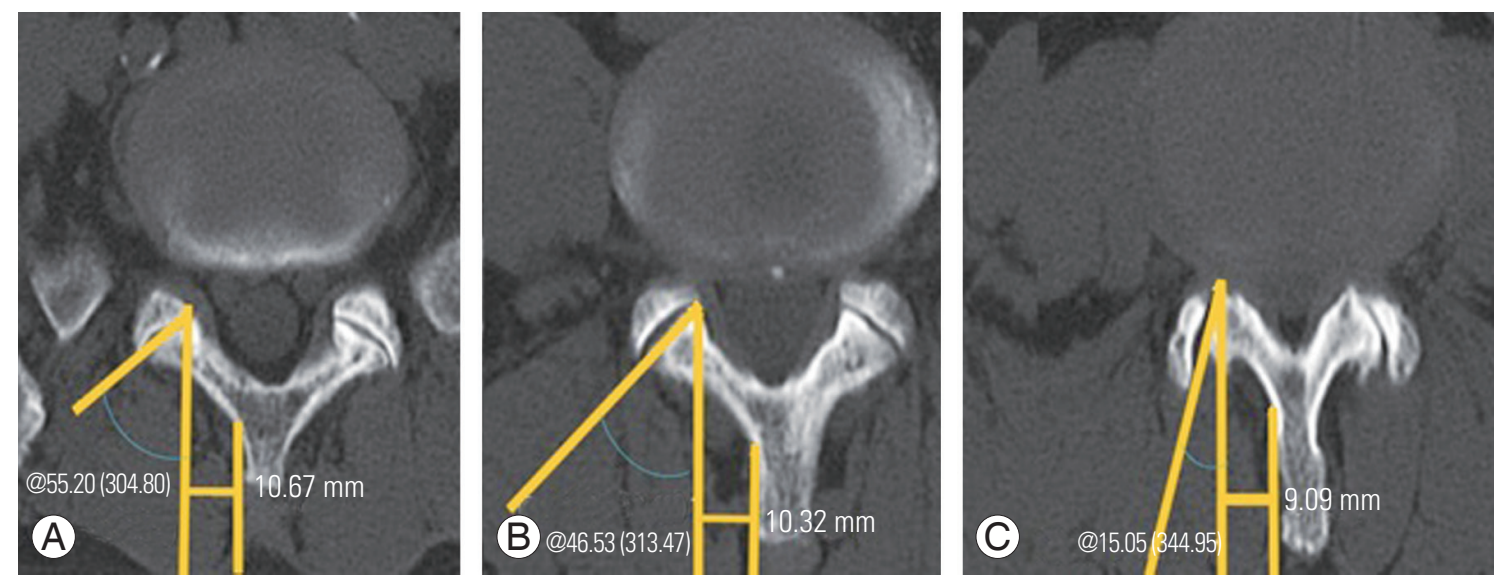

Fig. 2. (A-C) Computed tomography shows mean laminar sizes and facet angles in the lumbar stenosis patients. In this study, lamina size was $<11 \mathrm{~mm}$ from $\mathrm{L} 5$ to $\mathrm{L} 3$, with a steady decrease in facet angle from $\mathrm{L} 5$ to $\mathrm{L} 3$. 
of laminar size and facet angle measured in the lumbar stenosis, the lamina size (distance from spinous process to medial margin of facet) was $<11 \mathrm{~mm}$ for $\mathrm{L} 5$ and $9 \mathrm{~mm}$ for L3 with a steady decrease in facet angle from L5 to L3 (Fig. 2, Table 2). On the basis of these results, stenoscopy (H-View, Daejeon, Korea) was performed with an instrument having an $8.4 \mathrm{~mm}$ outer diameter, $5.7 \mathrm{~mm}$ working channel, and $12^{\circ}$ field of view, through which a $2-5 \mathrm{~mm}$ drill and bur and 1-5 mm Kerrison punch were used for laminectomy, foraminotomy, and discectomy (Fig. 3).

\section{Surgical procedure}

The procedure was performed with the patient under epidural anesthesia and placed in a prone, comfortable position on a radiolucent table. The incision location was confirmed by intraoperative fluoroscopy. After making a $7 \mathrm{~mm}$ vertical skin incision, a blunt dilator that served as a guide for the $9.5 \mathrm{~mm}$ outer diameter working sleeve

Table 2. Lamina size and facet angle in patients with lumbar stenosis; specification of stenoscopy

\begin{tabular}{lc} 
Characteristic & Value \\
Lamina size $(\mathrm{mm})(\mathrm{n}=50)$ & \\
\hline $\mathrm{L} 3$ & $9.09 \pm 1.75$ \\
\hline $\mathrm{L} 4$ & $10.32 \pm 3.37$ \\
\hline $\mathrm{L} 5$ & $10.67 \pm 3.20$ \\
\hline Facet angle $\left(^{\circ}\right)(\mathrm{n}=50)$ & \\
\hline L3-4 & $15.05 \pm 2.31$ \\
\hline L4-5 & $46.53 \pm 3.02$ \\
\hline L5-S1 & $55.20 \pm 3.52$ \\
\hline Specifications of stenoscopy & \\
\hline Outer diameter $(\mathrm{mm})$ & 8.4 \\
\hline Working channel $(\mathrm{mm})$ & 5.7 \\
\hline Optic view angle $\left({ }^{\circ}\right)$ & 12 \\
\hline
\end{tabular}

Values are presented as mean \pm standard deviation or number.
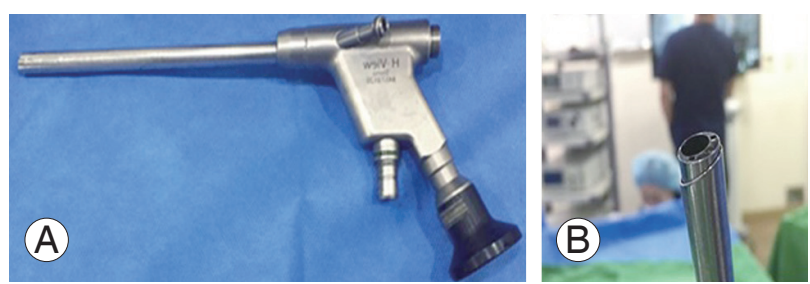

Fig. 3. (A, B) Percutaneous stenoscopic lumbar decompression set. The dimensions are $120 \mathrm{~mm}$ length, $8.4 \mathrm{~mm}$ outer diameter scope, 5.7 $\mathrm{mm}$ working channel, and a $12^{\circ}$ field of view. was advanced into the lamina on the ipsilateral side in a right-angle direction, just beside the spinous process. Subsequently, the working sleeve was inserted over the dilator and a rigid angle stenoscope $(8.4 \mathrm{~mm}$ outer diameter, $12^{\circ}$ view) was introduced into the lesion from one side through the working sleeve. The unique surgical approach through fatty atrophy between the spinous process and multifidus muscles helped to decrease the postoperative muscle-origin back pain and is considered an advantage of this process (Fig. 4). The epidural space was opened via laminectomy, and the ligamentum flavum and superior articular process were removed to expose the traversing root sequentially using a $4 \mathrm{~mm}$ drill and $5 \mathrm{~mm}$ Kerrison punch through the $5.7 \mathrm{~mm}$ working channel of the stenoscope. Laminotomy was performed to expose the uppermost portion of the ligamentum flavum, and as much of the ligamentum flavum was removed as possible. Following ipsilateral decompression, the contralateral ligamentous flavum and superior articular process were removed to decompress the contralateral traversing nerve root. Minimal bone work, as much as required, was performed
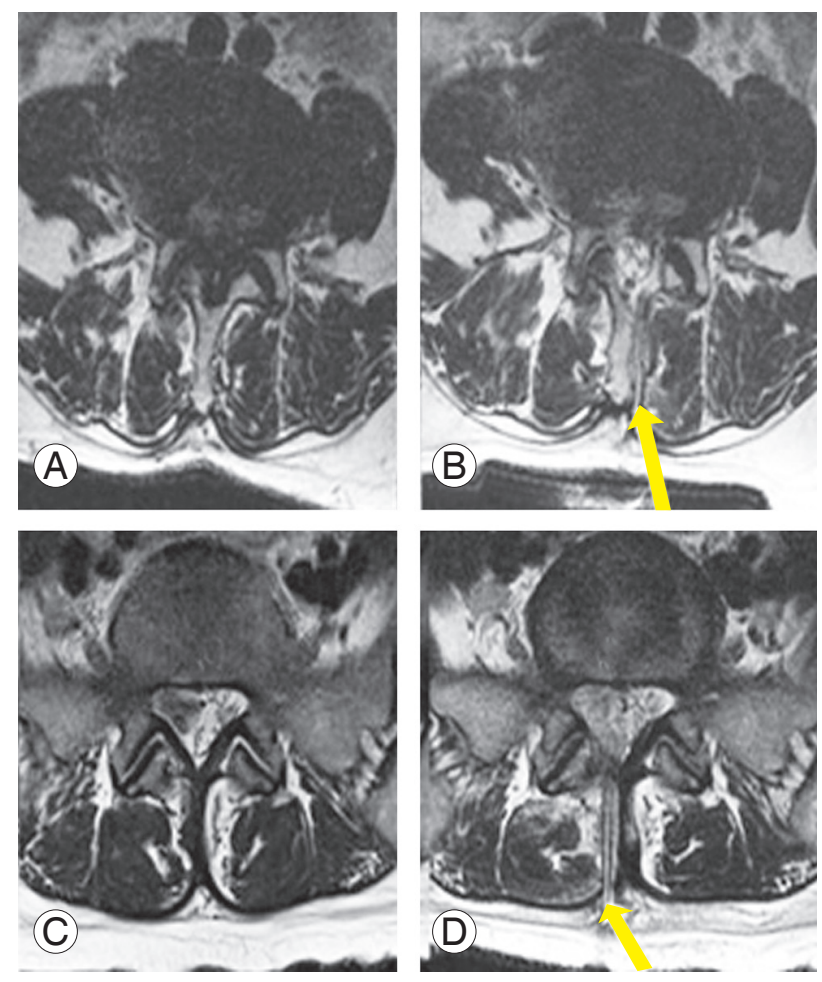

Fig. 4. Two cases of percutaneous stenoscopic lumbar decompression. (A, C) Preoperative MRI. (B, D) Postoperative MRI shows the entry point of approach, fatty atrophy between the spinous process and multifidus muscle ((arrow, postoperative drain). MRI, magnetic resonance imaging. 
to preserve the facet joint, which was the first priority (Fig. 5). The operative field was irrigated continuously with normal saline using the irrigation pump to provide a clean view with good visualization of epidural anatomy for safety purposes. In case of multiple level stenoses, decompression through a single skin entry was achieved via a special technique called the jumping technique (Fig. 6). In this technique, after completion of one level, the working sleeve was completely removed and moved cranially or caudally to the other target within the subcutaneous space under image guidance, but still within the same skin incision. Skin has good elasticity and can be used to make another muscle layer tract by subfascial dissection through a single incision. Then, the same process as for the PSLD procedure was performed in a different direction, upper or lower, for decompression of the remaining lumbar stenosis. After the first muscle tract, the subsequent two muscle tracts have an inconvenient approach angle, but do not interfere with decompression. After identification and confirmation of the interlaminar space and laminar space under the $\mathrm{C}$-arm, the dilator was introduced into the created path by a small forceps, and the working sleeve was inserted over the dilator. Upward and downward retraction of the skin allowed the tubular working sleeve to be placed in the upper and lower interlaminar spaces, where the decompression will be performed. Every step of the procedure was done under image intensifier control to confirm the exact entry point. The stenoscope was intro-
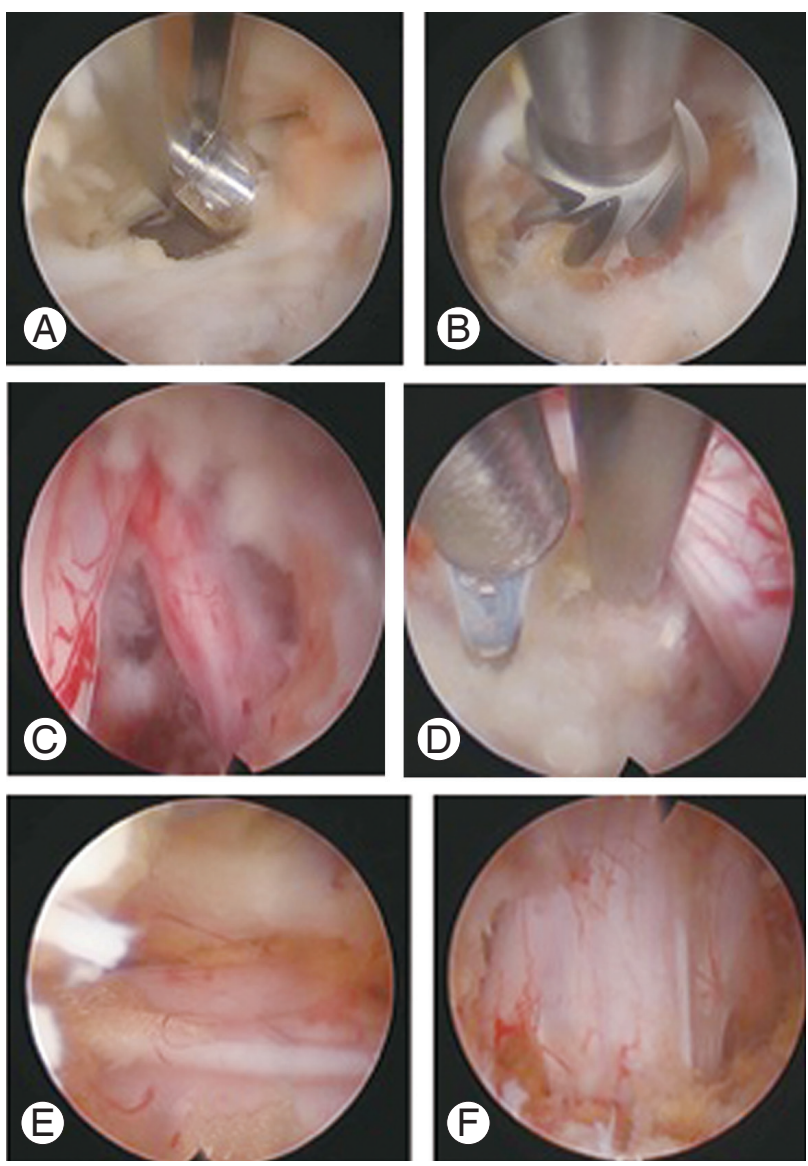

Fig. 5. Surgical technique. (A) Flavectomy with $5 \mathrm{~mm}$ Kerrison punch. (B) Bone work with $3 \mathrm{~mm}$ drill. (C) Axilla and shoulder of the exposed nerve root. (D) Nerve root retraction and annuloplasty. (E) Contralateral axilla and shoulder. (F) Endpoint of percutaneous stenoscopic lumbar decompression.
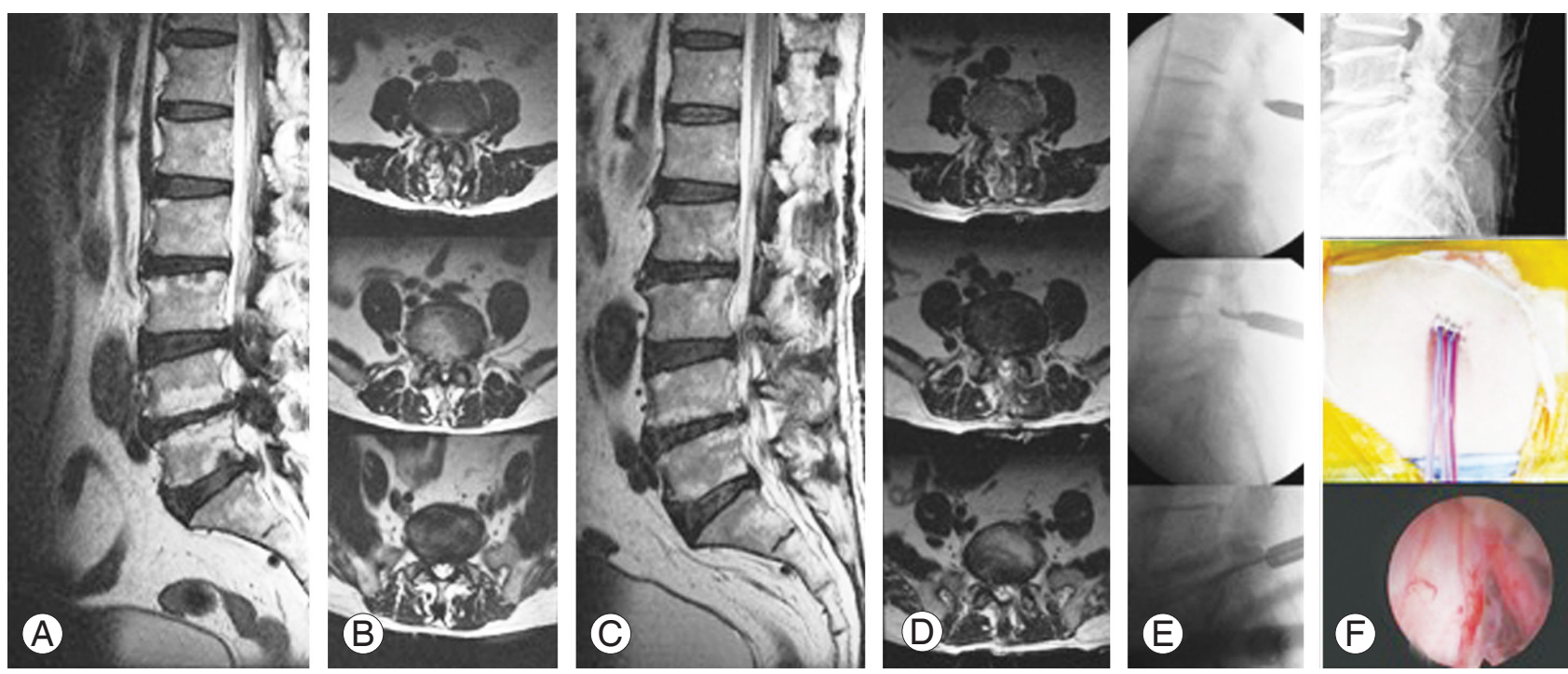

Fig. 6. L3-4, L4-5, L5-S1, and 3-level decompression with one skin incision by the jumping technique. (A, B) Preoperative MRI. (C, D) Postoperative MRI shows enough decompression without paraspinal muscle damage. (E) The jumping technique for multiple layer decompression with one skin incision. (F) Postoperative drainage and endpoint of percutaneous stenoscopic lumbar decompression. MRI, magnetic resonance imaging. 
duced into the lesion and subsequent procedures performed as described above. After the procedures, a drain was placed in the epidural space to prevent postoperative hematoma for 1 day (Fig. 6).

\section{Analysis of radiologic and clinical outcomes}

All patients underwent a postoperative MRI. Sagittal and
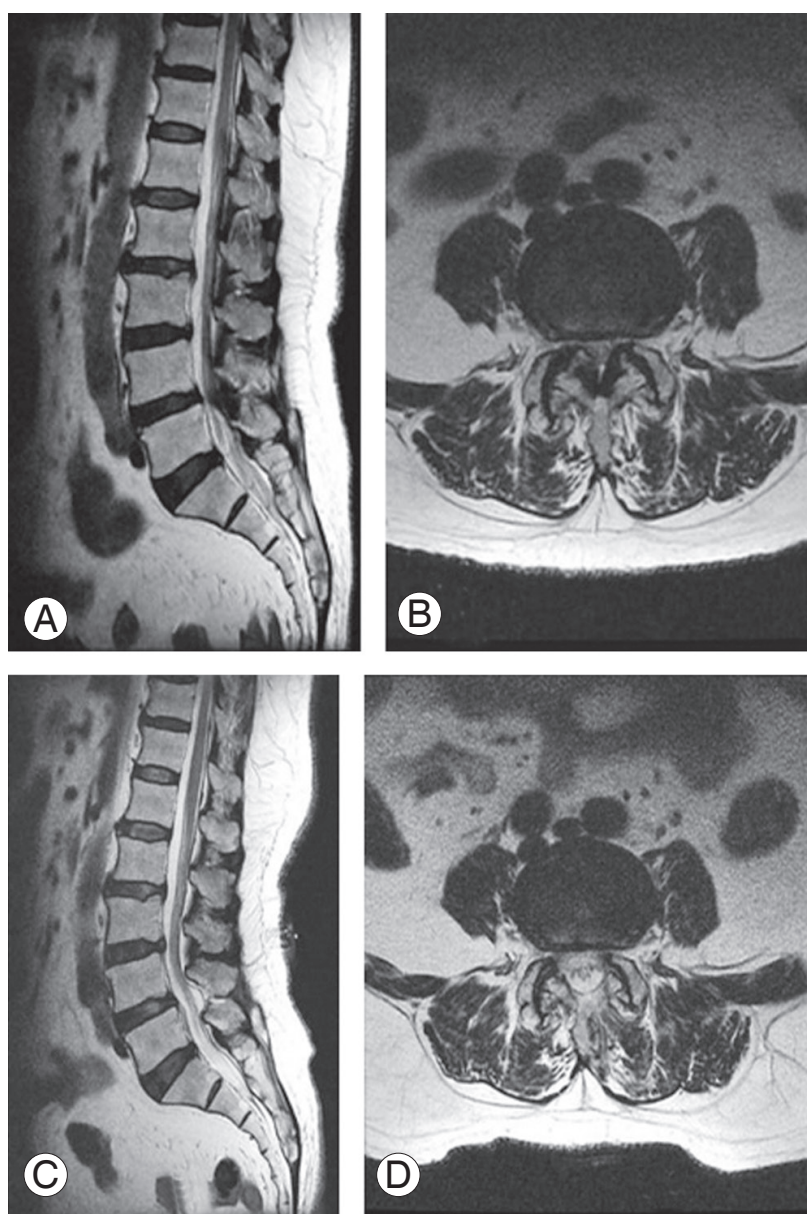

Fig. 7. L4-5 preoperative (A, B) and postoperative (C, D) magnetic resonance imaging. Full decompression with minimum bone work and no muscle injury are the advantages of percutaneous stenoscopic lumbar decompression (C, D). axial T2-weighted images on postoperative day 1 were compared with preoperative images to assess the changes in cross-sectional area (Fig. 7). Since the spinal canal is not uniformly cylinder, we divided it into three segments (upper, middle, and lower) at $6 \mathrm{~mm}$ intervals from upper to lower parts of the ligamentum flavum to determine the exact changes in cross-sectional area preoperatively and postoperatively. The methodology was designed to be as real as possible (Fig. 1). All patients were asked to complete a questionnaire regarding the VAS and ODI. VAS was checked preoperatively and postoperatively at 1 week and 3 and 12 months, and ODI was checked preoperatively and at 3 and 12 months postoperatively to analyze the clinical outcomes. Only 250 patients responded to the questionnaire.

\section{Results}

\section{Operative results}

Mean operating time was $32.3 \pm 15.25,64.4 \pm 37.3$, and $95.5 \pm 28.6$ minutes for bilateral decompression at 1,2 , and 3 levels, respectively. Vital signs during the procedures with epidural anesthesia were stable, and all patients had

Table 3. Mean operation time and duration of hospitalization

\begin{tabular}{ll} 
Variable & Value \\
\hline Operation time of bilateral decompression (min) & \\
\hline 1 Level & $32.3 \pm 15.25$ \\
\hline 2 Level & $64.4 \pm 37.3$ \\
\hline 3 Level & $95.5 \pm 28.6$ \\
\hline Duration of hospitalization (day) & \\
\hline 1 Level & $1.42 \pm 0.1$ \\
\hline 2 Level & $1.47 \pm 2.7$ \\
\hline L Level & $1.49 \pm 5.2$ \\
\hline
\end{tabular}

Values are presented as mean \pm standard deviation.

Table 4. Pre- and postoperative changes in cross-sectional area of lower, middle, and upper segment

\begin{tabular}{|c|c|c|c|c|}
\hline \multirow[b]{2}{*}{ Segment } & \multicolumn{2}{|c|}{ Mean cross-sectional area of canal } & \multirow[b]{2}{*}{$p$-value } & \multirow[b]{2}{*}{ Mean increased cross-sectional area (\%) } \\
\hline & Preoperative & Postoperative & & \\
\hline Lower & $161.4 \pm 3.2$ & $234.4 \pm 4.1$ & $<0.001$ & 45.3 \\
\hline Middle & $121.3 \pm 3.2$ & $206.9 \pm 3.6$ & $<0.001$ & 71.1 \\
\hline Upper & $125.9 \pm 3.6$ & $179.3 \pm 3.3$ & $<0.001$ & 39.7 \\
\hline
\end{tabular}

Values are presented as mean \pm standard deviation, unless otherwise stated. 
negligible blood loss with no clinical significance. Mean hospital stay was $1.42,1.47$, and 1.50 days, respectively. There was no significant difference in hospitalization between each group (Table 3).
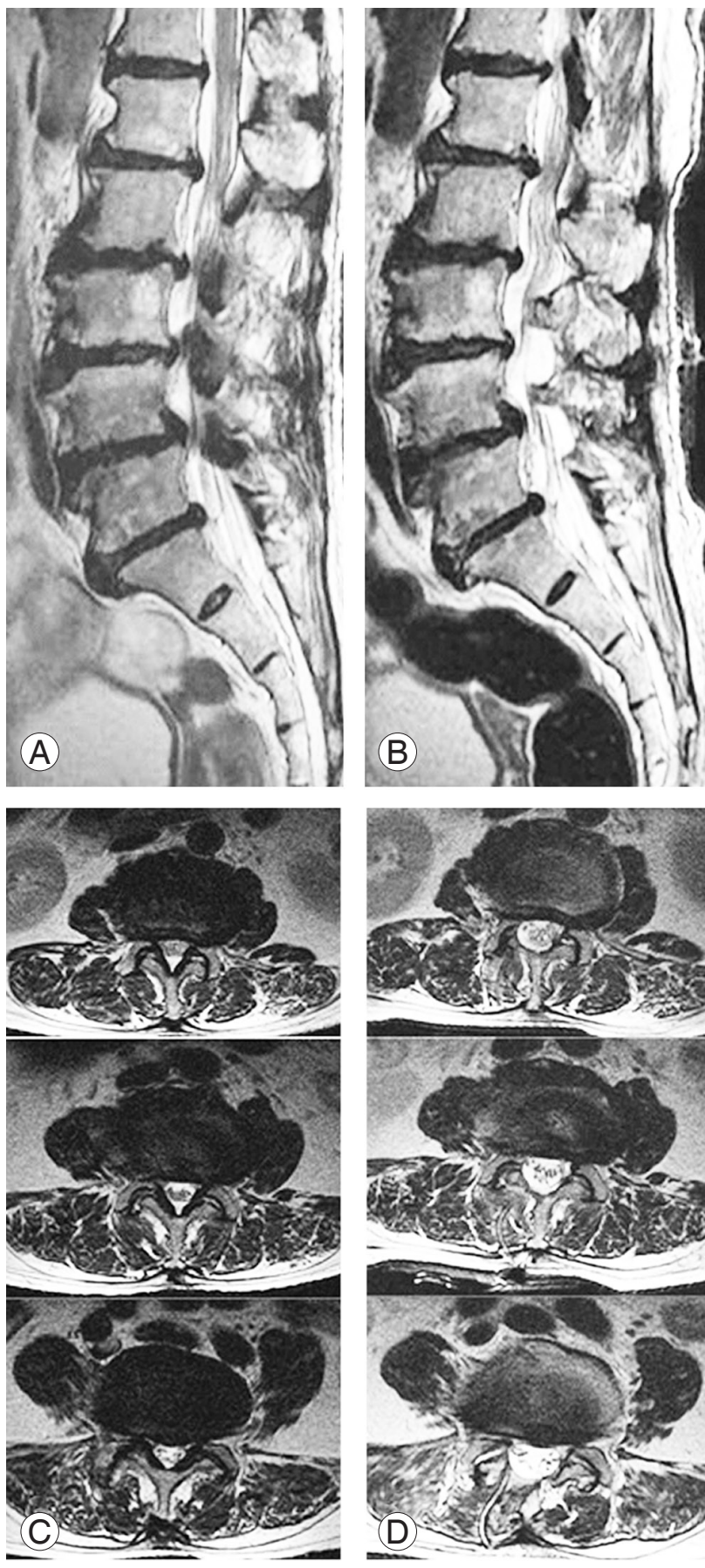

Fig. 8. L3-4, L4-5, L5-S1, and 3-level decompression with one skin incision. (A) Preoperative sagittal MRI. (B) Postoperative sagittal MRI. (C) Preoperative axial MRI. (D) Postoperative axial MRI. MRI, magnetic resonance imaging.

\section{Preoperative and postoperative change in cross- sectional area}

Postoperative MRI shows a statistically significant increase in cross-sectional area in each segment, with the largest increase in the middle segment (Table 4). There was no recognizable injury of multifidus muscle in any case, and no complications, including hematoma, were observed on postoperative MRI (Figs. 6-8).

\section{Clinical results}

The mean VAS score for back pain showed a statistically significant improvement from 6.24 preoperatively to 2.36 at 3 months for the 1-level and multiple-level decompression groups $(p<0.001)$. However, at 1 week, the VAS score for back pain was significantly higher in the multiple-level than in the 1-level decompression groups. The VAS score for leg pain improved significantly at 1 week and 3 months in both groups $(p<0.001)$. Mean ODI value improved from 60.6 preoperatively to 26.3 at 3 months postoperatively $(p<0.001)$. VAS and ODI at 12 months maintained considerable postoperative improvement $(p<0.001)$ (Table 5).

\section{Surgery-related complications}

Of the 450 patients, 13 (2.9\%) experienced symptomatic

Table 5. Patients pain assessment before and after surgery

\begin{tabular}{|c|c|c|c|}
\hline Clinical data & 1 Level & 2 Level & 3 Level \\
\hline \multicolumn{4}{|l|}{ VAS back } \\
\hline Preoperative & $6.00 \pm 4.21$ & $6.20 \pm 2.74$ & $6.52 \pm 4.02$ \\
\hline Postoperative $1 \mathrm{wk}$ & $2.70 \pm 4.14$ & $4.50 \pm 0.72$ & $4.60 \pm 0.62$ \\
\hline Postoperative 3 mo & $1.92 \pm 0.79$ & $2.51 \pm 1.32$ & $2.67 \pm 5.58$ \\
\hline Postoperative $12 \mathrm{mo}$ & $2.13 \pm 3.01$ & $2.24 \pm 4.02$ & $2.27 \pm 1.62$ \\
\hline \multicolumn{4}{|l|}{ VAS leg } \\
\hline Preoperative & $7.10 \pm 2.51$ & $7.20 \pm 5.03$ & $7.50 \pm 5.22$ \\
\hline Postoperative 1 wk & $2.31 \pm 7.21$ & $2.43 \pm 6.41$ & $2.47 \pm 2.73$ \\
\hline Postoperative 3 mo & $2.82 \pm 0.71$ & $2.86 \pm 3.91$ & $2.91 \pm 2.61$ \\
\hline Postoperative $12 \mathrm{mo}$ & $2.31 \pm 4.15$ & $2.40 \pm 0.79$ & $2.41 \pm 1.92$ \\
\hline \multicolumn{4}{|l|}{ Oswestry Disability Index } \\
\hline Preoperative & $58.81 \pm 11.65$ & $60.32 \pm 21.03$ & $62.72 \pm 21.92$ \\
\hline Postoperative 3 mo & $25.29 \pm 3.57$ & $26.63 \pm 32.11$ & $27.02 \pm 32.07$ \\
\hline Postoperative $12 \mathrm{mo}$ & $23.72 \pm 4.12$ & $24.72 \pm 2.76$ & $25.92 \pm 27.92$ \\
\hline
\end{tabular}

Values are presented as mean \pm standard deviation.

VAS, Visual Analog Scale. 
Table 6. postoperative complications

\begin{tabular}{lc} 
Complication & Value \\
\hline Incidental dural tear (root herniation) & $7(2)$ \\
Wound infection & 1 \\
\hline Epidural hematoma & 5 \\
\hline Reoperation & \\
\hline \multicolumn{1}{l}{ Percutaneous stenoscopic lumbar decompression } & 4 \\
\hline Open microscopic decompression & 2 \\
\hline
\end{tabular}

complications associated with PSLD. Four patients (0.9\%) required repeat PSLD within 6 weeks postoperatively due to incomplete decompression of the traversing root and postoperative hematoma. Dural tears during procedures were observed in seven patients (1.6\%) including two cases of root injury. Open microscopic laminectomy and fusion were done in two patients $(0.4 \%)$ due to rootlet herniation through the dural defect. There was no significant neurologic deficit of nerve root injury, but one patient had temporary leg dysesthesia. One patient with dural tears complained of a headache for 1 month postoperatively. Only one patient suffered from postoperative infection (Table 6). This patient was monitored for changes in the level of C-reactive protein and erythrocyte sedimentation rate, which are known as the most sensitive clinical laboratory markers to assess the presence of infection and effectiveness of treatment response. Also, MRI, the imaging modality of choice for diagnosis of postoperative infection, was analyzed. The patient's condition improved after management with broad-spectrum antibiotics and immobilization. There was no postoperative instability at the index segment as evaluated by dynamic radiographs at final follow-up.

\section{Discussion}

Our study demonstrated that PSLD can achieve sufficient decompression with a resultant significant reduction in pain and disability without leading to serious complications throughout postoperative follow-up. Although operative time increased according to the increase in operative disc levels, hospital stay was very similar regardless of the PSLD level. In a recent review article comparing parameters between MED and open laminectomy for spinal stenosis, Wong et al. [13] reported an average operative time for MED and open laminectomy of 124 and 101 minutes, respectively, and hospitalization of 0.75 and 3.27 days, respectively, with an overall complication rate of $7.9 \%$ and $16.1 \%$, respectively. The clinical outcome of PSLD appeared to be better compared with that of MED and open laminectomies, and shorter hospitalization and lower complication rates were observed when comparing PSLD and MED. It is hard to claim that these PSLD data may be comparable with other surgical modalities in different clinical studies. However, the comparative analysis could provide us with an idea about a new therapeutic modality and its potential in the surgical treatment of spinal stenosis. From our study, the importance of a multilevel PSLD procedure, the so-called jumping technique, becomes evident. To the best of our knowledge, this could be the first report regarding one-entry-multilevel endoscopic decompression for consecutive multilevel spinal stenosis worldwide. The advantages of the jumping technique might be a small incision with few skin wounds, which might be correlated with the smaller wound infection rate in our study. Fagundes et al. [14] recently reported that reduction in infection could be achieved not by using prophylactic antibiotics but by a minimal skin incision. In our study, irrespective of the number of levels operated, hospital stay was similar in all cases. Apparently, postoperative early recovery has no relation with the number of levels operated with PSLD.

Spinal stenosis, defined as a narrowing of the vertebral canal and/or the foramen causing compression of lumbosacral nerve roots, is the most common lumbar spine problem in people $>65$ years old [15]. Decompressive surgery for lumbar stenosis is the most common surgery for these patients [16]. Well-controlled clinical trials have shown statistically significant improvements in postoperative functional outcome and quality of life, compared with medical management [16]. Spinal stenosis usually begins with degeneration of the intervertebral disc and facet joints with resultant narrowing of the spinal canal and foramen [17]. Spinal stenosis is considered the last phase of the degenerative cascade as described by YongHing and Kirkaldy-Willis [18] in 1983. Depending on the range of hypermobility, the degree of instability varies from a first phase defined as 'micro-instability', to the second phase of overt instability, and to a third step of 'steno-instability.' The third stage must be the most stable among these three stages; however, the consequent development of the hypertrophic reaction of the ligamentum flavum and bony structures, including the facet joints, can 
lead to narrowing of the neural canal and foramen [18]. Therefore, a surgeon must be careful regarding intemperate removal of bony structures without special precaution, because such intemperate removal could cause reversion back to the earlier stage of degeneration and instability; in other words, causing recurrent instability. In this regard, the concept of preservation of bony structures including the facet joint should be upheld to avoid postoperative segmental instability [19].

In our study, only a small part of the superior facet process encroaching upon the lateral recess was removed; hence, the PSLD-operated cases demonstrated minimal facet removal. Another bony removal consisted of a small hole in the unilateral lamina of the endoscope to gain access to the epidural space. Eventually, most of the facet joint could be preserved even after PSLD, and this is one advantage of PSLD. According to our results, endoscopic laminectomy and foraminotomy appear to be safe and effective alternatives for surgical treatment of lumbar spinal stenosis in adults [20,21].

Open microscopic laminectomy or laminectomy has become a standard surgical management of degenerative lumbar stenosis. However, damage to the posterior ligamentum, muscles, and tissues remains unavoidable $[2,22,23]$. During the procedure, muscle detachment and dissection may cause muscle weakness and denervation, finally followed by atrophy, which is linked to an increase in the prevalence of post-spine surgery syndrome and chronic low back pain. Also, wide removal of facet joints results in potential complications of segmental instability, a long recovery time, and rehabilitation [24]. Endoscopic spine surgery can be a good alternative to open microscopic decompression, as this endoscopic technology is free from the aforementioned potential results. In our study, the preoperative and postoperative MRI findings revealed that PSLD preserved the normal structures apart from the proper discectomy and/or decompressed spinal stenosis. However, such results need more scientific evidence, such as information about preoperative and postoperative changes in paravertebral muscle volume measurement [25] and in tissue injury markers (serum enzymes) representing skeletal muscle injury. In our study, none of those factors were analyzed [26].

A new endoscopic system, stenoscopy, was designed in a surgeon-friendly manner to be used to achieve a translaminar or interlaminar approach to an epidural space lesion in the spinal canal and to perform decompression and/or discectomy in a safe and convenient manner. At 12 month postoperative follow-up, all clinical parameters, including changes in VAS and ODI (Table 5), demonstrated statistically significant improvement. Our patients had only canal and lateral recess stenoses. Therefore, postoperative change in the area and volume of the vertebral canal could be a parameter to assess quantitative and objective changes in the spinal canal width postoperatively compared with preoperative data. Our study was designed to measure the stenotic canal in the area and to compare the results to postoperative changes in the vertebral canal. Three cross-sectional slices were obtained from the index level. In each patient, a blinded investigator measured each slice and compared the preoperative and postoperative areas to evaluate the changes in volume using MRI. The spinal canal area measured postoperatively was significantly enlarged (Table 4). PSLD could lead to enlargement of the lumbar spinal stenosis postoperatively to a maximum of $71.1 \%$ in the middle segment of the treated canal at the index level and to $39.7 \%$ in the lower segment, but with minimal bone work. However, a high degree of canal enlargement is not considered better improvement of surgical outcome compared with a low degree of enlargement [27].

In our study, the overall complication rate was much lower than that for MED (2.9\% versus 7.9\%). Among the complications, durotomy occurred most frequently, but at a low rate of seven cases, $(53.8 \%$ of 13 complication cases; $1.9 \%$ of 450 total cases). Based on our experience, the incidence of dural tears appeared to increase when the superior articular process of the ipsilateral and contralateral side was resected. Intraoperatively, a thin layer of TachoSil, a hemostatic dura sealant, possibly might help repair the site of the dural tear and defects [28].

The reason for low overall complication rates might be that all five surgeons who performed the PSLDs had $>10$ years of clinical experience as a spine surgeon. There one case of infection might be an end result of quite a large amount of continuous saline irrigation (average 4,000 $\mathrm{mL}$ ) throughout each PSLD procedure. Also, a small single skin entry might be the reason for our extremely low infection. No patient demonstrated a significant neurological deficit. PSLD is expected to be used in patients with foraminal stenosis and all types of herniated discs at all spine levels. It is hypothesized that endoscopic spine surgery, such as PSLD, will replace most of the open or microscopic spine surgical procedures in the near future. 
Some limitations are associated with this study. First, this was a retrospective study, and it was difficult to control the bias and confounding variable. Second, there was no control group for comparison. Third, the follow-up was not long enough to conclude the surgical outcomes and complications. Because the initial benefits of surgical decompression might deteriorate with time [29], additional longer-term follow-up is needed. Also, future studies will provide an insight into the risk of postoperative instability and restenosis and the incidence of reoperation in a well-controlled multicenter study.

\section{Conclusions}

Our study demonstrated that full endoscopic decompressing procedures using PSLD have therapeutic feasibility in the surgical treatment of lumbar stenosis. PSLD achieved postoperative improvement, which was confirmed by the clinical follow-up and measurement of the postoperatively enlarged area, with minimal removal of medial facet joints and minimal damage in paraspinal muscles as evident by MRI. Monoportal PSLD has a potential for managing monolevel and multilevel spinal stenoses. PSLD results appeared compatible with those of conventional surgical treatment. Accordingly, PSLD could be an alternative to microscopic decompression or MED in the surgical management of lumbar stenosis along with some potential advantages, such as shorter hospital stays, earlier return to the workplace, and lower infection rate. Prospective randomized clinical trials are strongly warranted.

\section{Conflict of Interest}

No potential conflict of interest relevant to this article was reported.

\section{References}

1. Costa F, Sassi M, Cardia A, et al. Degenerative lumbar spinal stenosis: analysis of results in a series of 374 patients treated with unilateral laminotomy for bilateral microdecompression. J Neurosurg Spine 2007;7:579-86.

2. Rosen DS, O'Toole JE, Eichholz KM, et al. Minimally invasive lumbar spinal decompression in the elderly: outcomes of 50 patients aged 75 years and older. Neurosurgery 2007;60:503-9.
3. Marawar SV, Madom IA, Palumbo M, et al. Surgeon reliability for the assessment of lumbar spinal stenosis on MRI: the impact of surgeon experience. Int J Spine Surg 2017;11:34.

4. Gunzburg R, Szpalski M. The conservative surgical treatment of lumbar spinal stenosis in the elderly. Eur Spine J 2003;12 Suppl 2:S176-80.

5. Jackson RP, McManus AC, Moore J. Lumbar spinal stenosis: treatment options for an aging population. Mo Med 2012;109:466-9.

6. Arnoldi CC, Brodsky AE, Cauchoix J, et al. Lumbar spinal stenosis and nerve root entrapment syndromes: definition and classification. Clin Orthop Relat Res 1976;(115):4-5.

7. Demirayak M, Sisman L, Turkmen F, et al. Clinical and radiological results of microsurgical posterior lumbar interbody fusion and decompression without posterior instrumentation for lateral recess stenosis. Asian Spine J 2015;9:713-20.

8. Giannadakis C, Hammersbøen LE, Feyling C, et al. Microsurgical decompression for central lumbar spinal stenosis: a single-center observational study. Acta Neurochir (Wien) 2015;157:1165-71.

9. Nerland US, Jakola AS, Solheim O, et al. Minimally invasive decompression versus open laminectomy for central stenosis of the lumbar spine: pragmatic comparative effectiveness study. BMJ 2015;350:h1603.

10. Sakane M. Anatomical relationship between Kambin's triangle and exiting nerve root. Mini-Invasive Surg 2017;1:99-102.

11. Birjandian Z, Emerson S, Telfeian AE, Hofstetter CP. Interlaminar endoscopic lateral recess decompression-surgical technique and early clinical results. J Spine Surg 2017;3:123-32.

12. Hudak EM, Perry MW. Outpatient minimally invasive spine surgery using endoscopy for the treatment of lumbar spinal stenosis among obese patients. J Orthop 2015;12:156-9.

13. Wong AP, Smith ZA, Lall RR, Bresnahan LE, Fessler RG. The microendoscopic decompression of lumbar stenosis: a review of the current literature and clinical results. Minim Invasive Surg 2012;2012:325095.

14. Fagundes RB, Cantarelli JC Jr, Fontana K, Motta GL. Percutaneous endoscopic gastrostomy and peristomal infection: an avoidable complication with the use of a minimum skin incision. Surg Laparosc Endosc Percutan Tech 2011;21:275-7. 
15. Haddadi K, Ganjeh Qazvini HR. Outcome after surgery of lumbar spinal stenosis: a randomized comparison of bilateral laminotomy, trumpet laminectomy, and conventional laminectomy. Front Surg 2016;3:19.

16. Gibson JN, Waddell G. Surgery for degenerative lumbar spondylosis: updated Cochrane review. Spine (Phila Pa 1976) 2005;30:2312-20.

17. Fast A, Robin GC, Floman Y. Surgical treatment of lumbar spinal stenosis in the elderly. Arch Phys Med Rehabil 1985;66:149-51.

18. Yong-Hing K, Kirkaldy-Willis WH. The pathophysiology of degenerative disease of the lumbar spine. Orthop Clin North Am 1983;14:491-504.

19. Park MK, Kim KT, Cho DC, Sung JK. Surgical tips to preserve the facet joint during microdiscectomy. J Korean Neurosurg Soc 2013;54:366-9.

20. Ruetten S, Komp M, Merk H, Godolias G. Full-endoscopic anterior decompression versus conventional anterior decompression and fusion in cervical disc herniations. Int Orthop 2009;33:1677-82.

21. Ruetten S, Komp M, Merk H, Godolias G. Recurrent lumbar disc herniation after conventional discectomy: a prospective, randomized study comparing full-endoscopic interlaminar and transforaminal versus microsurgical revision. J Spinal Disord Tech 2009;22:122-9.

22. Asgarzadie F, Khoo LT. Minimally invasive operative management for lumbar spinal stenosis: overview of early and long-term outcomes. Orthop Clin North
Am 2007;38:387-99.

23. Hong SW, Choi KY, Ahn Y, et al. A comparison of unilateral and bilateral laminotomies for decompression of L4-L5 spinal stenosis. Spine (Phila Pa 1976) 2011;36:E172-8.

24. Sihvonen T, Herno A, Paljarvi L, Airaksinen O, Partanen J, Tapaninaho A. Local denervation atrophy of paraspinal muscles in postoperative failed back syndrome. Spine (Phila Pa 1976) 1993;18:575-81.

25. Ntilikina Y, Bahlau D, Garnon J, et al. Open versus percutaneous instrumentation in thoracolumbar fractures: magnetic resonance imaging comparison of paravertebral muscles after implant removal. J Neurosurg Spine 2017;27:235-41.

26. Kim KT, Lee SH, Suk KS, Bae SC. The quantitative analysis of tissue injury markers after mini-open lumbar fusion. Spine (Phila Pa 1976) 2006;31:712-6.

27. Ko S. Correlations between sedimentation sign, dural sac cross-sectional area, and clinical symptoms of degenerative lumbar spinal stenosis. Eur Spine J 2018;27:1623-8.

28. Ulivieri S, Peri G, Tiezzi G, Mileo E, Giorgio A, Oliveri G. From surgery to neurosurgery: our experience on the efficacy of fleece-bound sealing (TachoSil) for dural repair. G Chir 2014;35:195-8.

29. Atlas SJ, Deyo RA, Keller RB, et al. The Maine Lumbar Spine Study, part III: 1-year outcomes of surgical and nonsurgical management of lumbar spinal stenosis. Spine (Phila Pa 1976) 1996;21:1787-94. 